
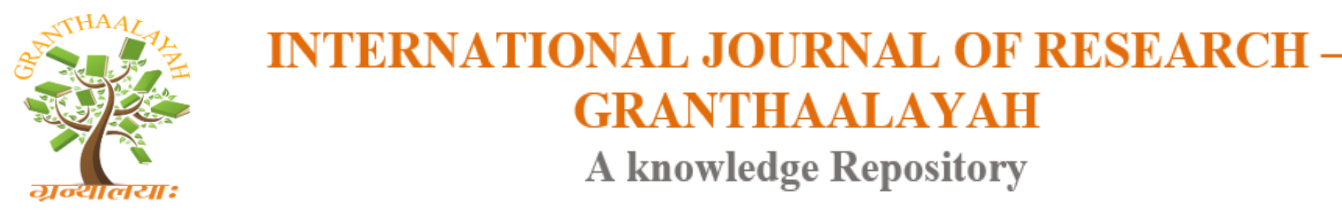

Science

\title{
EXTRACELLULAR BIOSYNTHESIS OF SILVER NANOPARTICLES USING FUNGUS PENICILLIUM SPECIES
}

\author{
Tejal Barkhade *1 \\ ${ }^{* 1}$ School of Nanosciences, Central University of Gujarat, Gandhinagar, India
}

\begin{abstract}
Synthesis of nanoparticles by biological process is good alternative as compare to physical and chemical processes because it's very easy, non-toxic, eco-friendly, and cheap. Biological synthesis combines biological principles (i.e., reduction/oxidation) by microbial enzymes. The advantages of fungal-mediated biosynthesis of nanoparticles have turned the attention of scientists to the kingdom of fungi. The present research study shows the biological synthesis of silver nanoparticles (AgNPs) by using filamentous fungus Penicillium sp. The biomass was harvested after 72 hours of growth by sieving through a plastic sieve and from this biomass cell filtrate was obtained for the synthesis process. The synthesis process was quite fast; when silver ion was interact with cell filtrate, within few minutes. After 24 hour of incubation period in dark condition, colorless cell filtrate solution with AgNO3 solution was turns in to brown color solution; that means synthesis process of silver nanoparticles was confirmed due to color change reaction. The characterization of synthesized Silver nanoparticles was done by Transmission Electron Microscopy (TEM) and Scanning Electron Microscopy (SEM), UV-Visible spectroscopy.
\end{abstract}

Keywords: Biosynthesis; Silver Nanoparticles (Agnps); Penicillium Sp.; Fungus; Characterization.

Cite This Article: Tejal Barkhade. (2018). "EXTRACELLULAR BIOSYNTHESIS OF SILVER NANOPARTICLES USING FUNGUS PENICILLIUM SPECIES.” International Journal of Research - Granthaalayah, 6(1), 277-283. 10.29121/granthaalayah.v6.i1.2018.1615.

\section{Introduction}

Nanomaterials (NMs) are defined as materials with at least one external dimension in the size range from approximately 1-100 nano meters. A focused integration of bio and nano techniques for biological synthesis of NMs, known as bio nanotechnology, has emerged from nanotechnology (Kaushik $\mathrm{N}$ et al 2010). Recent studies on the use of microorganisms in the synthesis of nanoparticles or nanomaterials are a relatively new and exciting area of research with considerable potential for development. The current research worldwide on the use of microorganisms like bacteria, algae, yeast, actinomycetes and fungi in the biosynthesis of metal nanoparticles achieved a major role and great success (Monaliben Shah et al 2015). 
Biological synthesis combines biological principles (i.e., reduction/oxidation) by microbial enzymes with physical and chemical approaches to produce nano-sized particles. Nanoparticles (NPs) are solid particles with all three external dimension at the nano scale that can drastically modify physicochemical properties compared to the bulk material. It can explicate actions depending on the chemical composition, biological actions, size and shape. Since, nanomaterials show large surface area to volume ratio; it finds wide range of applications in industries. The chemical and physical methods have a low productivity, non-eco-friendly, capital intensive and toxic. Therefore, biological synthesis either extracellular or intracellular from higher plants or microbes has gained an upper hand (Mandal D 2006).

\subsection{Fungi in Nanoparticles Synthesis}

To achieve the objective of developing simple and eco-friendly technology researchers in this field have turned to biological systems. Sastry and co-workers have demonstrated that other than prokaryotic organisms, such as bacteria, eukaryotic organisms like fungi can play an efficient role as living manufactoriese for the synthesis of different inorganic nanoparticles. Metal nanoparticles like gold, silver and platinum have gained considerable attention in recent times due to their fundamental and technological interest (M. Sastry et al 2004). My co nanotechnology is the interface between 'Mycology' and 'Nanotechnology' and has considerable potential, partly due to the wide range and diversity of the fungi. When focusing on the synthesis of nanoparticles using fungi, it was observed that nanoparticles of good monodispersity and well dimensions could be synthesized. As fungi are found to secrete high amount of protein they might result in the significant mass productivity of nanoparticles. The fungal proteins are capable of hydrolyzing metal ions. In addition to this, fungi are easy to isolate and culture.

Moreover, the downstream processing and the handling of fungal biomass are less complex than the synthetic methods. Recently, the utilization of biological systems, especially fungi, has emerged as a novel method for the synthesis of nanoparticles. Currently, there is a growing need to using environmentally friendly nanoparticles that do not produce toxic wastes in their process synthesis protocol. Certain bacteria, yeasts and now fungi play an important role in remediation of toxic metals through reduction of the metal ions. For example, environmentally friendly microorganisms could minimize the toxicity in the process of metallic nanoparticles production by reduction of the metal ions or by formation of insoluble complexes with metal ions (e.g. metal sulfides) in the form of colloidal particles. People have reported that fungus Verticillium sp. and Fusarium oxysporum, when exposed to gold and silver ions, reduced the metal ion fairly rapidly and formed respective metallic nanoparticles (Kuber C et al 2006, A. Ahmad al 2003).

\section{Materials and Methods}

The aim of the study was to synthesize silver nanoparticles by using filamentous fungus Penicillium sp. The fungus culture was isolated from e-waste contaminated soil sample collected from e-waste site, and maintained on potato dextrose agar (PDA) medium at $28^{\circ} \mathrm{C}$. The isolated fungus was identified using morphological characteristics and microscopic structure. 


\subsection{Isolation of Fungal Culture}

Isolation of soil fungi was performed by serial dilution and spread plate method. One gram of soil sample was serially diluted in sterilized distilled water to get a concentration range from 101 to $10-6$. A volume of $0.1 \mathrm{ml}$ of each dilution was transferred aseptically to PDA plates. The sample was uniformly distributed by using a sterile glass spreader. The plates were incubated at $28^{\circ} \mathrm{c}$ for 3 days. The fungal isolates were further sub cultured on the Potato Dextrose Agar (PDA) plates in order to obtain pure culture. Pure isolates were maintained at $4^{\circ} \mathrm{C}$ in refrigerator for further studies.

\subsection{Colony Characterization}

The fungal isolates were observed using hand lens and the colony morphology was identified with respect to color, shape, size, texture and nature of colony.

\subsection{Microscopic Characterization}

Fungal isolates were microscopically characterized by Lactophenol Cotton Blue mounting. The cell morphology was recorded with respect to spore chain morphology, hyphae and mycelium structure.

\subsection{Biomass Preparation}

The Penicillium sp.was selected for the synthesis of silver nanoparticles. The pure colony of isolated fungal species was inoculated in Potato dextrose broth $(200 \mathrm{ml})$ liquid media. The flask was incubated at $25^{\circ} \mathrm{C}$ for 3 days in a rotary orbital shaker at a speed of $150 \mathrm{rpm}$. The biomass was harvested after $72 \mathrm{~h}$ of growth. The biomass was washed with sterilized distilled water to remove any medium component. $20 \mathrm{~g}$ of biomass (fresh weight) was mixed with $200 \mathrm{ml}$ of deionized water in a $500 \mathrm{ml}$ Erlenmeyer flask and agitated in the same condition for $72 \mathrm{~h}$ at $25^{\circ} \mathrm{C}$. After the incubation, the cell filtrate was obtained by passing it through Whatman filter paper no.1.Filtrate was collected and used further for AgNPs synthesis.

\subsection{Fungal Mediated Synthesis of Silver Nanoparticles}

For the synthesis of silver nanoparticles, $10 \mathrm{ml}$ of $5 \mathrm{mM} \mathrm{AgNO} 3$ solution was mixed with $100 \mathrm{ml}$ of cell filtrate in a $250 \mathrm{ml}$ Erlenmeyer flask and incubated $28^{\circ} \mathrm{C}$ in dark for $24 \mathrm{hrs}$. Control (without the silver ion, only biomass) was also run along with the experimental flask. After 24 hour of incubation period in dark condition, colorless cell filtrate solution with AgNO3 solution was turns in to brown color solution; that means synthesis process of silver nanoparticles was confirmed due to color change reaction. The reduction of silver ion was confirmed by qualitative testing of supernatant by UV-visible spectrophotometer. Absorbance of sample supernatant was measured by using UV-visible spectrophotometer between 400-600 nm. Then AgNPs were concentrated by centrifugation of the reaction mixture at $6000 \mathrm{rpm}$ for 20 min thrice with distilled water and ethanol, and then kept it in hot air oven for drying. After drying nanoparticles were collected by scratching with the help of spatula and the sample was stored in a glass vial. After that, AgNPs were collected for further characterization. 


\subsection{Characterization of AgNPs}

Characterization of fungal mediated synthesized AgNPs were done by Particle Size Analyzer, UV-Visible spectroscopy, TEM and SEM.

\section{Result and Discussion}

\subsection{Morphological and Microscopic view of Penicillium sp.}

For synthesis of AgNPs, isolated and identified fungus Penicillium sp.was selected. Colony morphology appeared as velvety with initially white color and on aging green color colony on PDA medium and microscopic structure is shown in Fig.1

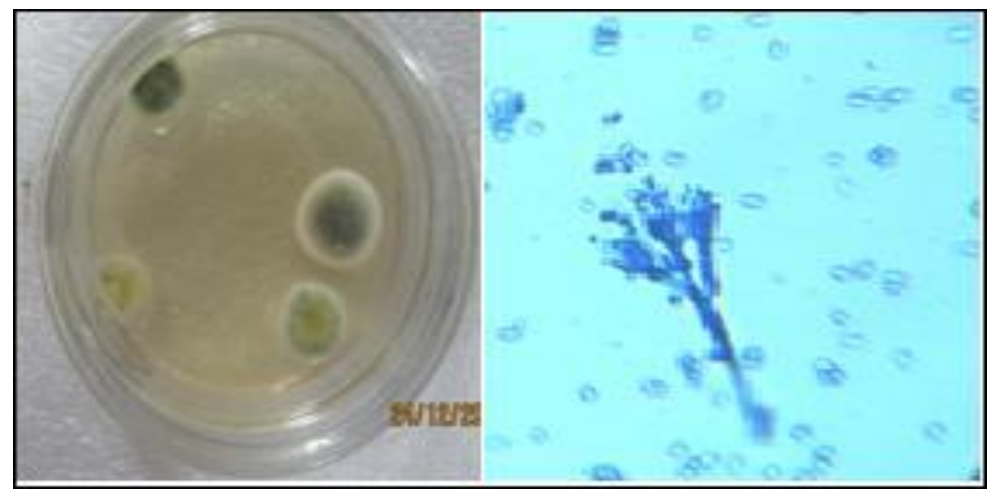

Figure 1: Morphological and Microscopic view of Penicillium sp.

\subsection{Biosynthesis of AgNPs}

The pure colony of Penicillium sp. transfer to potato dextrose broth, the potato dextrose broth with colony was kept in rotatory shaker at $150 \mathrm{rpm}$ for 3 days at $28^{\circ} \mathrm{C}$. After three days fungal biomass produced. Then fungal biomass washed properly with distilled water to remove remaining broth. Then after washing fungal biomass again kept in shaker with $200 \mathrm{ml}$ distilled water for 3 days. After incubation fungal biomass filtrate with filter paper. Cell filtrate was used for synthesis of silver nanoparticles (fig. 2).
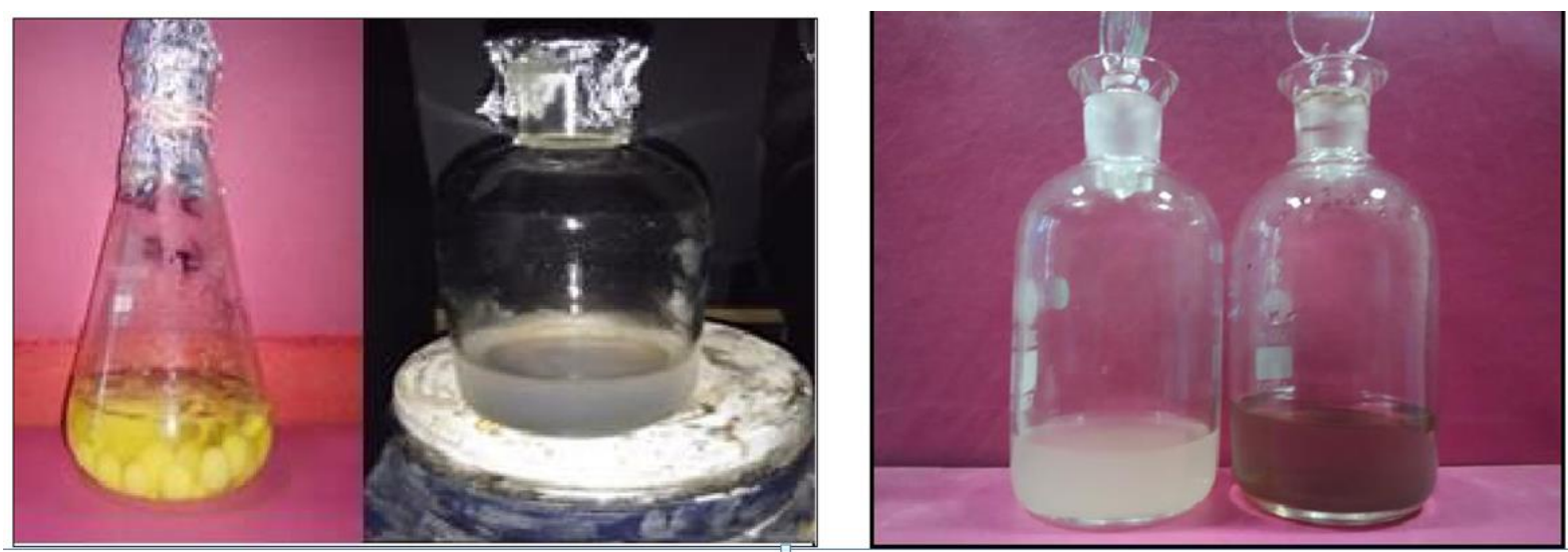

Figure 2: Fungal biomass and cell filtrate turnes into brown colour indicates synthesis of AgNPs 
The AgNO3 solution mixed in fungal cell filtrate and after that, the bottle of cell filtrate with AgNO3 solution kept in dark for 24 hours with control. After 24 hours of incubation in dark cell filtrate color changes to color less to brownish color (fig.3) it indicates that, the formation of silver nanoparticles was successfully done by using biological method for synthesis of AgNPs.

After completion of synthesis process of AgNPs, Silver nanoparticles were centrifuged three times by giving washing treatment with distilled water and ethanol, and then nanoparticles were kept in hot air oven for drying. Dry powder of silver nanoparticles were further characterized by TEM, SEM, PSA and UV-Visible spectroscopy.

\subsection{Characterization Techniques}

About $0.001 \mathrm{~g}$ of silver nanoparticles was dispersed in $20 \mathrm{ml}$ of distilled water and kept for 15-20 minutes for sonication and about 2-3 $\mathrm{ml}$ was taken in the cuvette, and reading was taken using Particles Size Analyses, Malvern (PSA) to analyze the average particle size and size distribution. The reduction of silver ions was confirmed by qualitative testing of supernatant by UV-visible spectrophotometer. $1 \mathrm{ml}$ of sample supernatant was withdrawn after 24 hours and absorbance was measured by using UV-visible spectrophotometer between 400-600 nm (fig.3). The absorbance peak was observed at $420 \mathrm{~nm}$.

For viewing TEM images the sample was dissolved in distilled water and sonicated for 10 minutes, and then a single drop of aqueous solution containing AgNPs was loaded on the copper grid and dried by allowing water to evaporate at room temperature. The grid containing silver nanoparticles s scanned by a Transmission Electron Microscope (TEM) 200 kV Technai-20, Phillips, and Holland. The confirmation of size and shape of AgNPs done by TEM. The size of AgNPs was observed 9-15 nm in TEM analysis. For observing shape and morphology of silver nanoparticles, Environment Scanning Electron Microscope (SEM) with EDAX Philips, Holland, ESEM TMP+EDAX was used. The size of nanoparticles was $25-35 \mathrm{~nm}$ was noted by SEM analysis (fig.4).

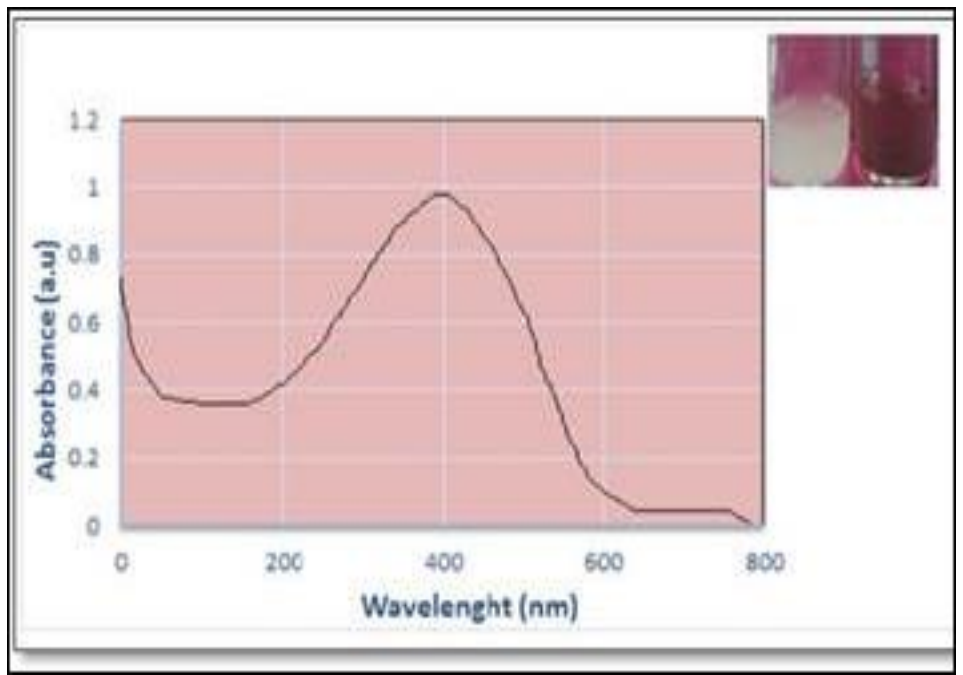

Figure 3: UV-Visible spectra of fungal mediated synthesized AgNPs 


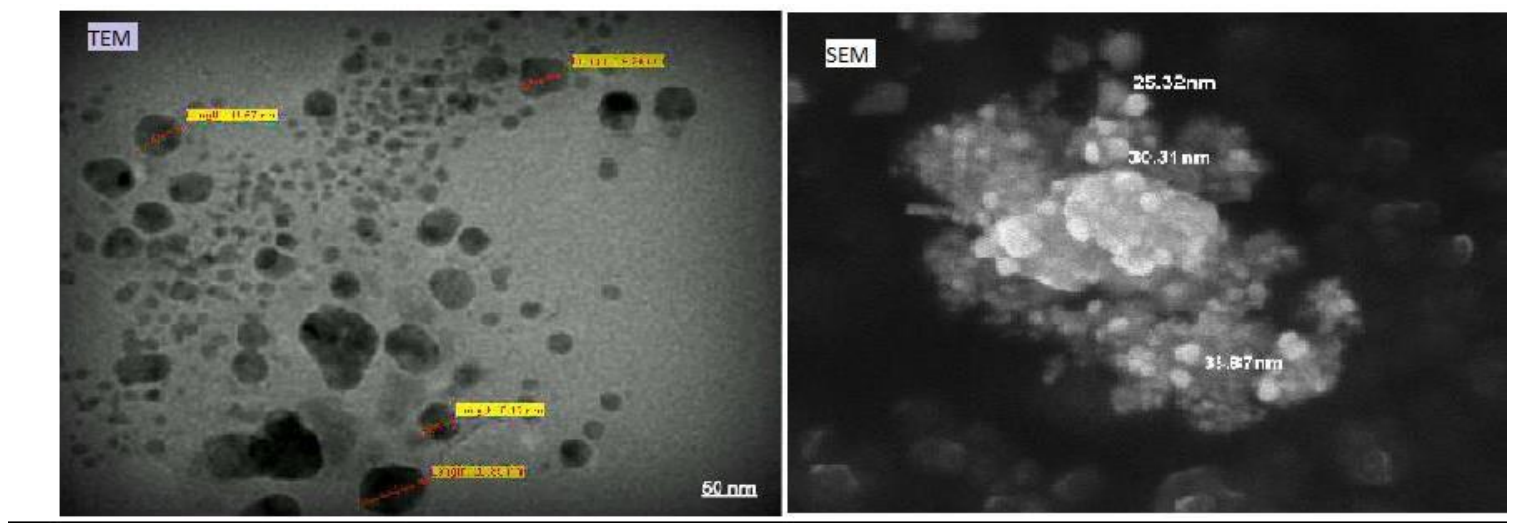

Figure 4: TEM \& SEM image of biological synthesized silver nanoparticles

\section{Conclusions}

Present study used a biological method for synthesis of silver nanoparticles because it was found that biological method is non- toxic, eco-friendly, cheap for synthesis of nanoparticles as compare to chemical and physical method. Fungal mediated synthesis of silver nanoparticles was done by fungus Penicillium sp. has good control over a size distribution of nanoparticles. This filamentous fungus species was isolated from soil sample. Fungal biomass cell filtrate was used for synthesis of AgNPs, because biomass was very easy to handle in laboratory and possess high wall-binding capacity, as well as intracellular metal uptake capabilities as compare to bacteria. Syntheses of silver nanoparticles were confirmed by UV-Visible spectroscopy and color change reaction of cell filtrate after 24 hours. Biologically synthesized AgNPs were characterized by TEM, SEM, for size shape purpose. Which gave very good size of nanoparticles between 9$15 \mathrm{~nm}$ sizes.

\section{Acknowledgements}

First and foremost, I offer my sincere gratitude to my supervisor, friends, and family. I am thankful to UGC, New Delhi for providing fellowship like RGNF for financial support.

\section{References}

[1] Kaushik N. Thakkar, MS, Snehit S. Mhatre, MS, Rasesh Y. Parikh, MS (2010)"BIOLOGICAL SYNTHESIS OF METALLIC NANOPARTICLES” Nanomedicine: Nanotechnology, Biology, and Medicine" 6, 257-262 https://doi: 10.1016/j.nano.2009.07.002. E pub 2009 Jul 16.

[2] Monaliben Shah, Derek Fawcett, Shashi Sharma, Suraj Kumar Tripathy and Gérrard Eddy Jai Poinern (2015) "GREEN SYNTHESIS OF METALLIC NANOPARTICLES VIABIOLOGICAL ENTITIES" Materials 2015, 8, 7278-7308;https:// doi:10.3390/ma8115377

[3] Mandal D, Bolander, M E, Mukhopadhyay, D, Sarkar, G, Mukherjee, P, ( 2006) "THEUSE OF MICROORGANISMS FOR THE FORMATION OF METAL NANOPARTICLES AND THEIR APPLICATION". Applied Microbiology and Biotechnology, 69, 485-49 https://doi.org/10.1007/s00253-005-0179-3

[4] A. Ahmad, S. Senapati, M.I. Khan, R. Kumar, M. Sastry, (2003) "EXTRACELLULAR BIOSYNTHESIS OF MONODISPERSED GOLD NANOPARTICLES BY A NOVEL EXTERMOPHILLIC ACTINOMYCETE, THERMONOSPORASP." Langmuir 19, 35503553.https://doi.org/: 10.1021/la0267721 
[5] kuber C. Bhainsa, S.F. D'Souza (2006) “EXTRACELLULAR BIOSYNTHESIS OF SILVER NANOPARTICLES USING THE FUNGUS ASPERGILLUS FUMIGATES" Colloids and Surfaces B: Biointerfaces 47 160-164. https:// doi:10.1016/j.colsurfb.2005.11.026

[6] A. Ahmad, P. Mukherjee, S. Senapati, D. Mandal, M.I. Khan, R. Kumar,M. Sastry, (2003) "EXTRACELLULAR BIOSYNTHESIS OF SILVER NANOPARTICLES USING THE FUNGUS FUSARIUM OXYSPORUM" Colloids Surf. B 28, 313318.https://doi.org/10.1016/S0927-7765 (02)00174-1

*Corresponding author.

E-mail address: tejalbarkhade04@ gmail.com 